\title{
THE CYCLING OF MATERIAL BETWEEN THE SOLAR CORONA AND CHROMOSPHERE
}

\author{
N. Guerreiro ${ }^{1}$, Viggo Hansteen ${ }^{1}$, and B. De Pontieu ${ }^{2}$ \\ ${ }^{1}$ Institute of Theoretical Astrophysics, University of Oslo, P.O. Box 1029 Blindern, N-0315 Oslo, Norway; n.m.r.guerreiro@astro.uio.no \\ ${ }^{2}$ Lockheed Martin Solar \& Astrophysics Laboratory, Org. A021S, Building 252, 3251 Hanover Street, Palo Alto, CA 94304, USA \\ Received 2012 October 22; accepted 2013 April 4; published 2013 May 3
}

\begin{abstract}
Observations of transition region emission lines reveal the presence of redshifts in lines formed from the top of the chromosphere up to temperatures of about $2.5 \times 10^{5} \mathrm{~K}$ and blueshifts for temperatures above that. However, it is doubtful that the apparent large downward flows in the lower transition region represents an emptying of the corona, so some mechanism must be responsible for maintaining the mass balance between the corona and the lower atmospheric layers. We use a three-dimensional magnetohydrodynamics code to study the cycling of mass between the corona, transition region, and chromosphere by adding a tracer fluid to the simulation in various temperature intervals in the transition region. We find that most of the material seen in transition region emission lines formed at temperatures below $3 \times 10^{5} \mathrm{~K}$ is material that has been rapidly heated from chromospheric temperatures and thereafter is pushed down as it cools. This implies that the bulk of transition region material resides in small loops. In these loops, the density is high and radiative cooling is efficient.
\end{abstract}

Key words: Sun: chromosphere - Sun: corona - Sun: transition region

\section{INTRODUCTION}

The source for the heating of the corona has been a hot topic for over half a century, but still remains poorly understood even though the basic scenario seems clear (Mariska 1992): given the large "mechanical" energy reservoir of the convection zone, the coronal heating problem can be split into two parts, i.e., transporting the energy flux generated in the convection zone into the corona and, thereafter, in thermalizing this energy flux. After it was shown observationally (Athay \& White 1978) that heating of the corona through the dissipation of acoustic waves is not possible, there have been two main scenarios for coronal heating, both involving the magnetic field. These can be loosely characterized as "AC," e.g., wave heating, and "DC," e.g., nanoflare models. AC heating is constrained by the difficulty on the one hand of transporting fast mode waves into the corona, and on the other by the low dissipation rates of "Alfvén" or Alfvénictype waves. The DC heating models are constrained by the low coronal resistivity and therefore by the necessity of structuring the field on very small scales in order to build up the needed large gradients in the coronal magnetic field.

During the past years the nano-flare coronal heating theory has received considerable support from numerical studies of the braiding process (Galsgaard \& Nordlund 1996; Hendrix et al. 1996) and from "realistic" three-dimensional (3D) magnetohydrodynamics (MHD) models of the photosphere-corona system (Gudiksen \& Nordlund 2005; Hansteen et al. 2007; Bingert $\&$ Peter 2011). These models show that the nano-flare scenario originally envisioned by Parker (1972), where the magnetic field is stressed by photospheric granular motions, does indeed lead to a $1.0 \times 10^{6} \mathrm{~K}$ corona which reproduces many of the coronal observables (Peter et al. 2006; Hansteen et al. 2010). The numerical models imply the presence of intense dissipative events throughout the chromosphere and corona that peak near the transition region where the current dissipation per particle is highest (Hansteen et al. 2010; Bingert \& Peter 2011). As a corollary, the numerical models also predict that the presence of redshifts in the upper chromosphere and lower transition region are due to episodic heating events (Peter et al. 2006; Hansteen et al. 2010), and in addition, that the redshifts correspond to a net downward velocity throughout the lower transition region.

The net redshifts observed in transition region lines has long been known to pervade all emission up to $5.0 \times 10^{5} \mathrm{~K}$ (Doschek et al. 1976; Brekke et al. 1997; Chae et al. 1998; Curdt et al. 2008). At greater temperatures Solar and Heliospheric Observatory/Solar Ultraviolet Measurement of Emitted Radiation observations indicate that net blueshifts often are found (Peter \& Judge 1999). A large number of models have been put forward attempting to explain the redshifts in the transition region: Pneuman \& Kopp (1977), Athay \& Holzer (1982), and Athay (1984) claim the redshifts are caused by the downflow of previously heated spicular material. Bradshaw \& Cargill (2010) have recently constructed models based on a similar scenario. Hansteen (1993) showed that downwardly propagating compressive waves, e.g., generated by nano-flares in the corona, could lead to a net redshift. Spadaro et al. (2006) used onedimensional models of coronal loops to infer that redshifts at temperatures under $10^{5} \mathrm{~K}$ could eventually result from transient heating near the foot points. Peter et al. (2006) first found redshifts from a similar mechanism arising in 3D numerical simulations spanning the photosphere to the corona, but did not go into detail as to why a net redshift was obtained. In a recent follow up based on these models, Zacharias et al. (2009) suggested that the redshifts are signatures of cooling gas draining from the corona after the reconnection events resulting from the disconnection of loops.

However, even if the redshifts pervade the lower transition region and suggest the presence of downflows throughout (Foukal 1976; Dammasch et al. 2008), the corona does not drain over time and its mass does not necessarily decrease. In fact, we find that in our simulations (Hansteen et al. 2010) the coronal mass actually increases or remains constant with time, even while a net mass flux downward through the transition region is recorded during the entire simulation run. This strange set of affairs is shown in Figure 1 which records the evolution of the B1 model of Hansteen et al. (2010) which comprises an area of $16.6 \times 8.3 \mathrm{Mm}^{2}$ and a corona some $8 \mathrm{Mm}$ high. The average mass flux through two surfaces, one defined by a 

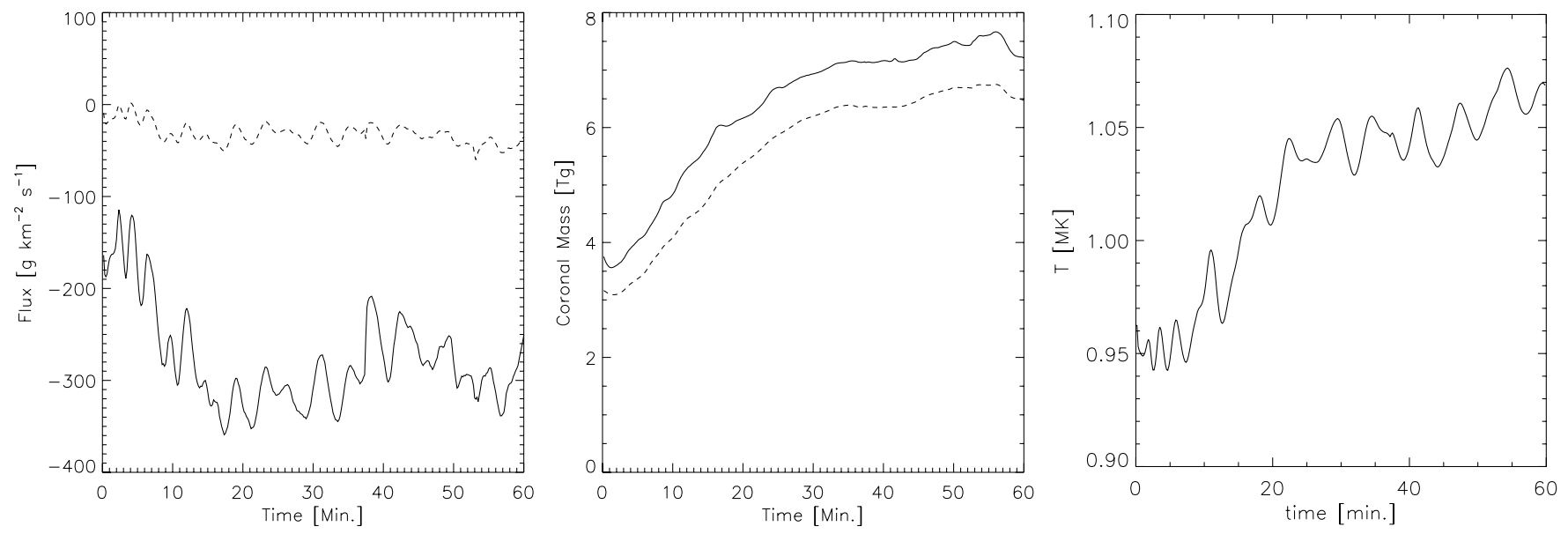

Figure 1. Left panel: simulated mass loss through surfaces at $T=2 \times 10^{4} \mathrm{~K}$ (solid line) and $T=3 \times 10^{5} \mathrm{~K}$ (dashed line). Middle panel: total coronal mass including all material with temperature above $T=2 \times 10^{4} \mathrm{~K}$ (solid line) and $3 \times 10^{5} \mathrm{~K}$ (dashed line). Right panel: average temperature of all material above $2 \times 10^{4} \mathrm{~K}$. Data taken from simulation B1 of Hansteen et al. (2010).

temperature of $T=2.0 \times 10^{4} \mathrm{~K}$, the other by a temperature of $T=3.0 \times 10^{5} \mathrm{~K}$, are plotted as functions of time in the left panel. There is a net downward mass flux through both surfaces, of some $-3 \times 10^{-9} \mathrm{~g} \mathrm{~cm}^{-2} \mathrm{~s}^{-1}$ for the $T=3.0 \times 10^{5} \mathrm{~K}$ surface and roughly ten times that for the surface defined by $T=2.0 \times 10^{4} \mathrm{~K}$. Thus, more than $13 \mathrm{Tg}$ transition region material flows downward through the surface defined by $T=3.0 \times 10^{5} \mathrm{~K}$ during the one hour simulation run. The middle panel shows the evolution of the total coronal mass above these layers: in spite of the large downflow, the mass of material above $T=3.0 \times 10^{5} \mathrm{~K}$ increases from a total of $3 \mathrm{Tg}$ in the first 30 minutes by more than $3 \mathrm{Tg}$ and thereafter remains roughly constant at $6.5 \mathrm{Tg}$. The net downward flux at the base of the transition region at $2.0 \times 10^{4} \mathrm{~K}$ is larger by a factor 10 , while the total mass in the interval between $2.0 \times 10^{4} \mathrm{~K}$ and $3.0 \times 10^{5} \mathrm{~K}$ remains nearly constant. The right panel of Figure 1 shows the time evolution of the average temperature of all material above $2 \times 10^{4} \mathrm{~K}$. Note that this time evolution is as much as one would expect for a model where the coronal mass balance is set by chromospheric evaporation (Klimchuk 2006; Reale 2010). A robust model capable of explaining the plasma cycling between the corona and the lower atmospheric layers for the quiet Sun depends upon its ability to reproduce the strong redshifts in the transition region and at the same time explain how coronal mass is retained (or grows). Explaining the contradiction between the mass loss through plasma flows and the more or less constant coronal mass is the aim of this paper, which we hope will shed light on the coronal mass budget and the interchange of mass between corona, transition region, and chromosphere.

We insert a tracer fluid into the transition region of the B1 model originally described by Hansteen et al. (2010) in order to follow the dynamic evolution of this vigorously heated plasma. In particular, we seek to describe the dynamics of a plasma layer initially found in the temperature interval, $\left[2.0 \times 10^{4}, 3.0 \times 10^{5}\right] \mathrm{K}$, as it evolves as a result of episodic heating, thermal conduction, radiative cooling, and mass flows through the transition region. We also present a limited number of examples of plasma flow along field lines for low loops that mainly contain transition region material and for higher loops where the apex temperatures are greater.

A short description of the code used and the methodology used to construct the simulation is presented in Section 2, and a discussion of the initial model is presented in Section 3. Results are shown and discussed in Section 4, while the conclusions follow in Section 5.

\section{NUMERICAL METHODS AND MODEL}

\subsection{The Bifrost MHD Code}

The initial model of the convection zone to corona simulation described here, as well as the cycling of transition region mass, is modeled using the Bifrost code (Gudiksen et al. 2011). This code is designed to solve the equations of MHD in the context of the upper convection zone and solar atmosphere, including the photosphere, chromosphere, transition region, and corona:

$$
\begin{gathered}
\frac{\partial \rho}{\partial t}=-\nabla \cdot(\rho \mathbf{u}) \\
\frac{\partial \rho \mathbf{u}}{\partial t}=-\nabla \cdot(\rho \mathbf{u u}-\tau)-\nabla P+\mathbf{J} \times \mathbf{B}+\rho \mathbf{g} \\
\mu \mathbf{J}=\nabla \times \mathbf{B} \\
\mathbf{E}=\eta \mathbf{J}-\mathbf{u} \times \mathbf{B} \\
\frac{\partial \mathbf{B}}{\partial t}=-\nabla \times \mathbf{E} \\
\frac{\partial e}{\partial t}=-\nabla \cdot(e \mathbf{u})-P \nabla \cdot \mathbf{u}+\mathbf{Q} .
\end{gathered}
$$

Here, $\rho$ represents the mass density, $\mathbf{u}$ the velocity vector, $P$ the gas pressure, $\mathbf{J}$ the electric current density vector, $\mathbf{B}$ the magnetic field, $\mathbf{g}$ the gravitational acceleration, $\mathbf{E}$ the electric field vector, and $e$ the internal energy. The magnetic diffusivity, viscous stress tensor, and vacuum permeability are written as $\eta$, $\tau$, and $\mu$, respectively. The variable $\mathbf{Q}$ in the energy equation contains the terms and processes needed to model the outer solar atmosphere. For the experiment described here these include non-gray radiative transfer including scattering for the photosphere and lower chromosphere (Hayek et al. 2010), an approximation to the non-LTE radiative terms that dominate the upper chromosphere (Carlsson \& Leenaarts 2012) and optically thin radiative losses in the transition region and corona. Thermal conduction along the magnetic field is a dominant term in the energy equation in the transition region and corona and is included implicitly through operator splitting. To relate the density and internal energy to the pressure and temperature 
an equation of state based on LTE ionization is tabulated and look up is performed via bi-linear interpolation. The lower boundary allows flows to exit unimpeded while the entropy of inflowing material is set to maintain the effective temperature at the solar value of some $T_{\text {eff }}=5.8 \times 10^{3} \mathrm{~K}$. At the upper boundary transparent boundary conditions are applied. Details of the implementation of these terms are discussed at length in Gudiksen et al. (2011).

\subsection{A Minority Fluid}

Bifrost is written in a modular and quite flexible manner, so adding extra equations is relatively straightforward. We have added an extra "minority" fluid species designed to mark and trace the dynamics of material originally in the transition region. The continuity equation used to follow the evolution of the tracer fluid is the same as that used for the density $\rho$, i.e., Equation (1). Note that this equation does not have any effects on the modeled MHD quantities but rather is passively carried around by the plasma velocity $\mathbf{u}$ :

$$
\frac{\partial \psi}{\partial t}+\nabla \cdot(\mathbf{u} \psi)=0
$$

where $\psi$ is defined to be the tracer fluid's density. The tracer fluid is placed in a certain volume of the solar atmosphere by specifying its location. This can be done in a number of different ways: it can either be set by defining a volume which lies inside a given height range, or by setting a pressure, or temperature interval in which the tracer material is placed. In the following we found it generally preferable to use a temperature interval to select the initial plasma volume meant to be tracked in our experiment.

The tracer is constructed such that $\psi$ is equal to the plasma density $\rho$ of each cell inside the volume we are interested in, and to $\psi=\rho \times 10^{-5}$ outside the specified volume, which we hereafter define as the background. In summary, for our experiment $\psi=\rho$ if $2.0 \times 10^{4} \mathrm{~K}<T<3.0 \times 10^{5} \mathrm{~K}$ and $\psi=\rho \times 10^{-5}$ outside this temperature range at $t=0 \mathrm{~s}$. Thereafter, the tracer fluid is allowed to evolve according to Equation (7).

Initially, the material traced is spread in the height interval [1.2-3.2] $\mathrm{Mm}$ and the density ranges between $10^{-12.5} \mathrm{~g} \mathrm{~cm}^{3}$ and $10^{-14.8} \mathrm{~g} \mathrm{~cm}^{3}$ as shown in Figure 2 .

Since there potentially are large gradients, both in the tracer fluid itself and between the tracer fluid and the background, a numerical "mass diffusion" term is added to the right hand side of Equation (7) using exactly the same methodology as described by (Gudiksen et al. 2011). Experimentation has shown that the value assigned to this term does not have any significant impact on the results reported here.

\section{A MODEL OF THE SOLAR ATMOSPHERE FROM CONVECTION ZONE TO CORONA}

We insert the tracer fluid into the model previously described in Hansteen et al. (2010): The computational box consists of a grid of $256 \times 128 \times 160$ points corresponding to a volume of $16.6 \times 8.3 \times 15.5 \mathrm{Mm}$ on the Sun, designed to stretch from the upper convection zone $1.5 \mathrm{Mm}$ below the photosphere into the corona, some $14 \mathrm{Mm}$ above. A uniform grid spacing of $65.0 \mathrm{~km}$ is chosen for the $x$ and $y$ directions, while for the $z$ direction a non-uniform grid was adopted to guarantee a proper resolution of the different layers of the solar atmosphere, i.e., the grid becomes sparser from the photosphere to the

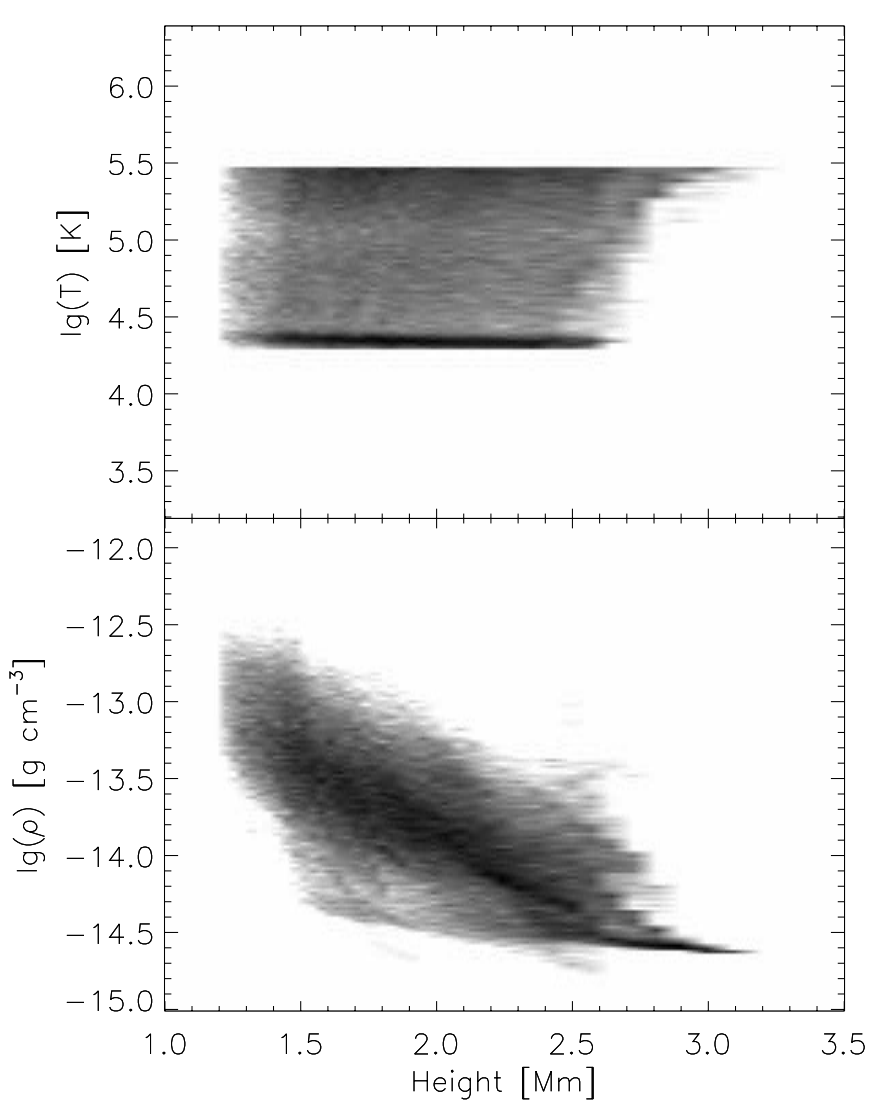

Figure 2. State of the tracked lower transition region plasma at $t=0 \mathrm{~s}$. Temperature (top) and density (bottom).

corona. The grid spacing is $\Delta z=32.0 \mathrm{~km}$ in the photosphere, $\Delta z=45.0 \mathrm{~km}$ at about $2 \mathrm{Mm}$ ( $\sim$ transition region) and increases to $\Delta z=441.0 \mathrm{~km}$ above $10 \mathrm{Mm}$ in the corona.

The code runs with periodic boundary conditions in the $x$ and $y$ directions, and non-periodic in the $z$ direction. The zero point of the $z$-scale is set to be approximately in the location where $\tau_{500}=1$, i.e., in the photosphere.

The energy losses through radiation in the photosphere and chromosphere are balanced by the introduction of an inflowing heat flux at the bottom boundary. Convective and photospheric motions generate acoustic waves that propagate into the chromosphere where, depending on the magnetic field configuration, they steepen and form shocks. Thus, the atmospheric layers just above the photosphere are energized by the passage and dissipation of acoustic shocks. At greater heights, where plasma $\beta<1$ and the magnetic field becomes more important, Joule heating dominates the heating of the atmosphere as the Poynting flux generated below is dissipated. This Poynting flux results from the braiding of magnetic field lines as described by Gudiksen \& Nordlund (2005), Hansteen \& Gudiksen (2005), Hansteen et al. (2007), and Bingert \& Peter (2011). In the model presented here there is also a certain amount of Poynting flux injected into the atmosphere as a result of flux emergence, as described by Martínez-Sykora et al. (2008, 2009), but the ultimate source of Poynting flux is not important to the main results of this paper.

The average temperature structure in this model is thus maintained by the fluid motions in the convection zone, by radiative transfer in the photosphere, by the balance between acoustic shocks and radiative losses in the lower chromosphere, and between Joule heating, thermal conduction, and radiative 

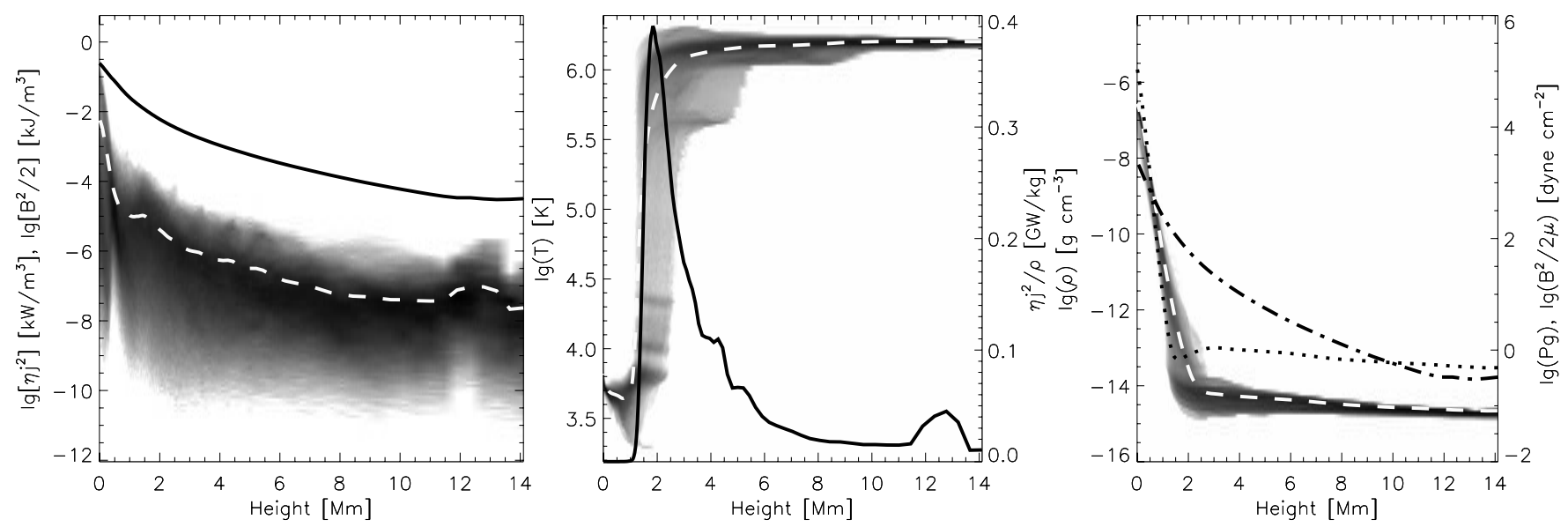

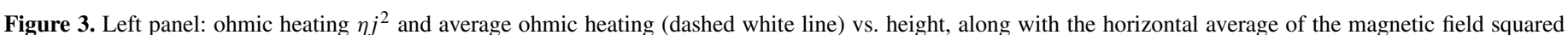

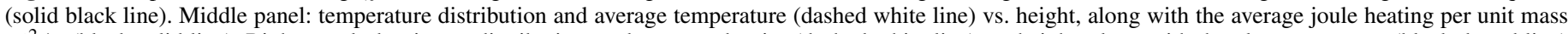

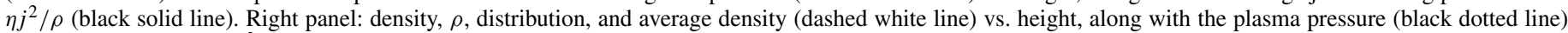
and magnetic pressure, $B^{2} / \mu$ (black dot-dashed line). The variables displayed show the state of the plasma at $t=0 \mathrm{~s}$.

losses in the upper chromosphere, transition region, and corona. The average structure of the atmosphere and of the Joule heating are shown in Figure 3. The average temperature shown in the middle panel decreases from the photosphere, at $0 \mathrm{Mm}$, to the bottom of the chromosphere where it reaches a minimum value of about $4410 \mathrm{~K}$ below $1 \mathrm{Mm}$. Thereafter, the temperature rises slowly through the upper chromosphere up to the bottom of the transition region, at $1.5 \mathrm{Mm}$, were a dramatic temperature increase occurs. We find coronal temperatures of the order of a million degrees or greater at heights of about $2.5 \mathrm{Mm}$. Overplotted is the average Joule heating per gram (per particle) which shows a clear maximum in the vicinity of the transition region. In the right panel the average magnetic pressure, $B^{2} / 2 \mu$, the gas pressure, $P_{g}$, and the density, $\rho$, for the initial model are displayed. The plasma density decreases from the top of the convection zone, $\rho \approx 10^{-5} \mathrm{~g} \mathrm{~cm}^{-3}$, to the corona $\rho \approx 10^{-15} \mathrm{~g} \mathrm{~cm}^{-3}$. The gas pressure decreases rapidly, from $10^{7}$ dyne $\mathrm{cm}^{-2}$ at the top of the convection zone to some 10 dyne $\mathrm{cm}^{-2}$ at the bottom of the transition region. On the other hand, the magnetic pressure decreases more smoothly from $B^{2} / 2 \mu_{0} \approx 10^{4}$ dyne $\mathrm{cm}^{-2}$ to values of the order $B^{2} / 2 \mu_{0} \approx$ 5 dyne $\mathrm{cm}^{-2}$. Note that, on average, $\beta>1$ in the convection zone up to some $0.6 \mathrm{Mm}$ above the photosphere, above this point the energy density of the magnetic field dominates the gas pressure.

A discussion of the distribution of the Joule heating in this model is also made in Hansteen et al. (2010). In that paper, it is concluded that the reason the heating per particle peaks in the transition region is that the scale height of Joule heating is of the same order as that of the magnetic field energy density $B^{2} / 2 \mu_{0}$ which is $\sim 600 \mathrm{~km}$. On the other hand, the pressure and density scale height of the chromosphere is of order only $200 \mathrm{~km}$ or less, and therefore the heating per particle grows exponentially until radiative losses from the (upper) chromosphere can no longer balance the Joule heating and the temperature rises. With rising temperatures the pressure scale height increases, rapidly becoming greater than that of Joule heating $(\sim 5000 \mathrm{~km})$ and the scale height of the Joule heating per particle is then set by the scale height of the magnetic energy density instead of the pressure or density scale height which is much greater, say some $50 \mathrm{Mm}$.

Figure 3 also shows that the temperature and density structure and the Joule heating per particle have a large spread in values.
Indeed, the Joule heating per particle varies by some five orders of magnitude, while the temperature varies by up to three orders of magnitude in the vicinity of the transition region, which in this model can be found from $1 \mathrm{Mm}$ above the photosphere up to $3 \mathrm{Mm}$ above the photosphere. Clearly, heating is concentrated in certain specific locations and as a result the upper atmosphere in this model of the Sun is quite inhomogeneous. The model has a minimum temperature of about $2.0 \times 10^{3} \mathrm{~K}$ and a maximum of about $1.9 \times 10^{6} \mathrm{~K}$ and a maximum and minimum density of about $10^{-6} \mathrm{~g} \mathrm{~cm}^{-3}$ and $10^{-15} \mathrm{~g} \mathrm{~cm}^{-3}$, respectively, at the beginning of the run.

Further insight into the spatial organization of the Joule heating is given in Figure 4 which shows the heating per particle in the computational box at the moment the tracer was introduced, as seen from the side and from above. Such vigorous heating events are episodic, last for about $100 \mathrm{~s}$, and, as noted above, are spatially dispersed, though concentrated near the transition region. Note that the locations of large heating per particle follow the structure of the magnetic field to a certain extent. Weaker components of heating appear to be more evenly distributed in space than regions of intense sporadic heating. The regions of high heating are short lived.

\section{RESULTS}

In model B1 of Hansteen et al. (2010) the average coronal heating rate is initially low, but increases some 20 minutes after the start of the simulation and remains high, though episodic, thereafter. We insert the tracer fluid during the latter, high heating, period at $t=30, t=50, t=55$ and $t=60$ minutes in order to allow for different start up settings especially in terms of the heating distribution. In each case we run the tracer fluid calculation for a duration of two minutes.

The initial set up of the tracer fluid, $t=0 \mathrm{~s}(60$ minutes into the B1 simulation), is shown in the left panel of Figure 5. The tracer fluid shown is originally comprised of some $0.7 \mathrm{Tg}$ of lower transition region plasma, defined as all the plasma in the temperature interval $\left[2 \times 10^{4}, 3 \times 10^{5}\right] \mathrm{K}$. The extent of the tracer fluid is indicated by the two purple isosurfaces. The lower of these is located at $z \approx 1.2 \mathrm{Mm}$, while the height of the upper layer is at $z \approx 3.3 \mathrm{Mm}$. The plasma of the lower transition region as delineated here forms a corrugated surface: its lowest points are found where the magnetic field is close 


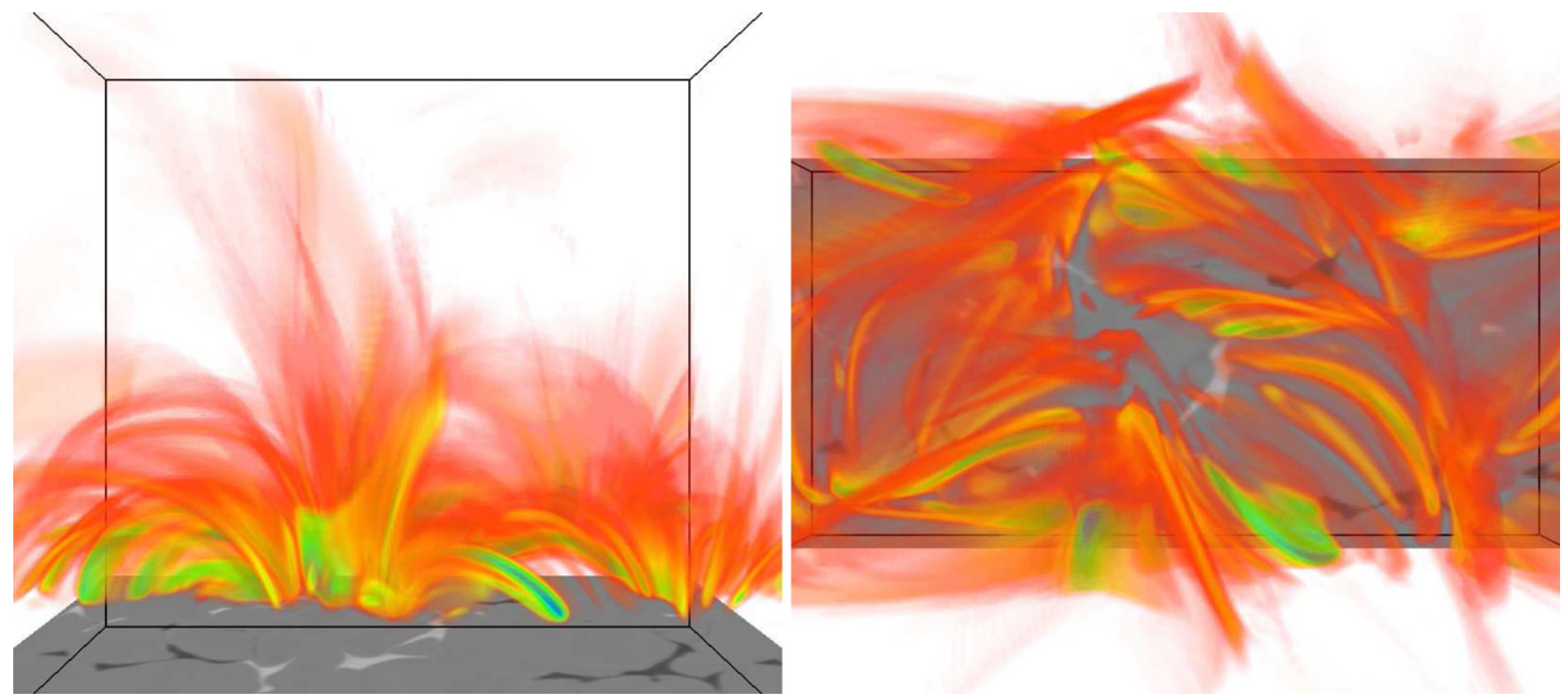

Figure 4. Current density squared per particle $\left(j^{2} / \rho\right)$ (side view left, top view right): red/yellow weakest, green/blue strongest. Low current density regions are given low opacity and therefore are transparent. The $B_{z}$ component of the magnetic field is shown in gray in the photosphere at $z=0 \mathrm{Mm}$, where $\tau_{500} \sim 1$.
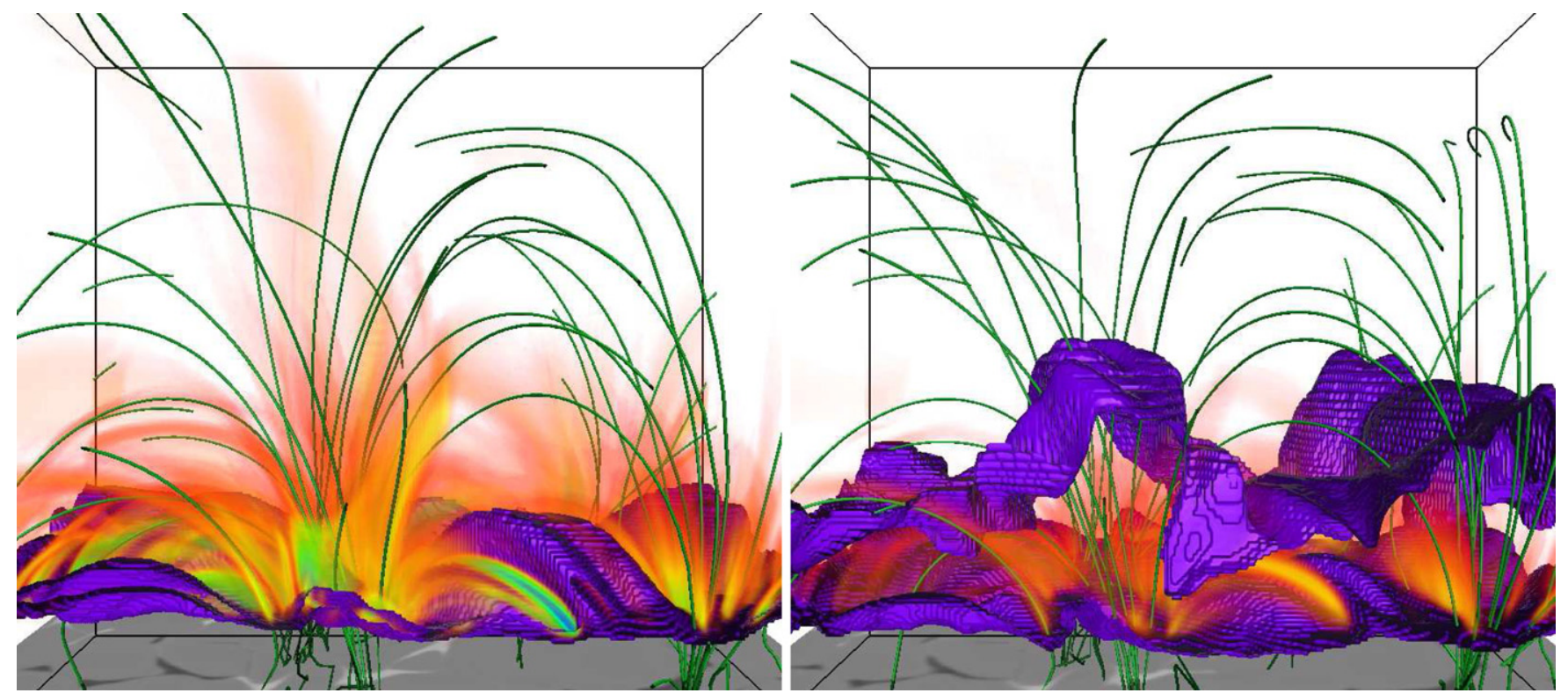

Figure 5. Left panel: volume of the tracer fluid in the temperature interval $\left[2.0 \times 10^{4}, 3.0 \times 10^{5}\right] \mathrm{K}$, at $t=0 \mathrm{~s}$ is enveloped by the purple isocontours. Right panel: evolutionary state of the isocontours after 2 minutes. In both panels, some magnetic field lines (green lines) and the current density (same color scale as Figure 4) are displayed.

to vertical, and reaches its greatest heights where the field is nearly horizontal. The transition region surface is immersed in several regions of intense heating, where the heating per particle $\left(j^{2} / \rho\right)$ is high. Individual elements of intense heating roughly follow the magnetic field lines and thereby stretch highest in locations where the field is close to vertical, but regions of high heating per particle also occurs where the magnetic field is nearly horizontal.

The right panel of Figure 5 shows the situation after 120 s: The upper isosurface showing the top layer of the fluid originally at $3 \times 10^{5} \mathrm{~K}$ has risen several thousand kilometers upward into the corona, especially in regions where the field is close to vertical, reaching heights of greater than $7 \mathrm{Mm}$. We also find a significant height increase in the regions where the field is mostly horizontal. The movement of the bottom layer, originally at $2 \times 10^{4} \mathrm{~K}$ is not discernible from this figure, but we shall see in the following that in fact most of the mass loss from the lower transition region is downward, cooling to chromospheric temperatures. Note also that the intense heating per particle evident in the left panel is reduced somewhat at the time the simulation is finished $120 \mathrm{~s}$ later.

Consider now the global evolution of this lower transition region layer, as well as an upper transition region layer initialized with plasma in the temperature range $\left[3 \times 10^{5}, 7 \times 10^{5}\right] \mathrm{K}$. In Figure 6 we show as a function of time the total amount of traced material that cools below the originally defined temperature (dashed line), the amount of traced material that is heated to temperatures above the original range (dot-dashed line) as well 


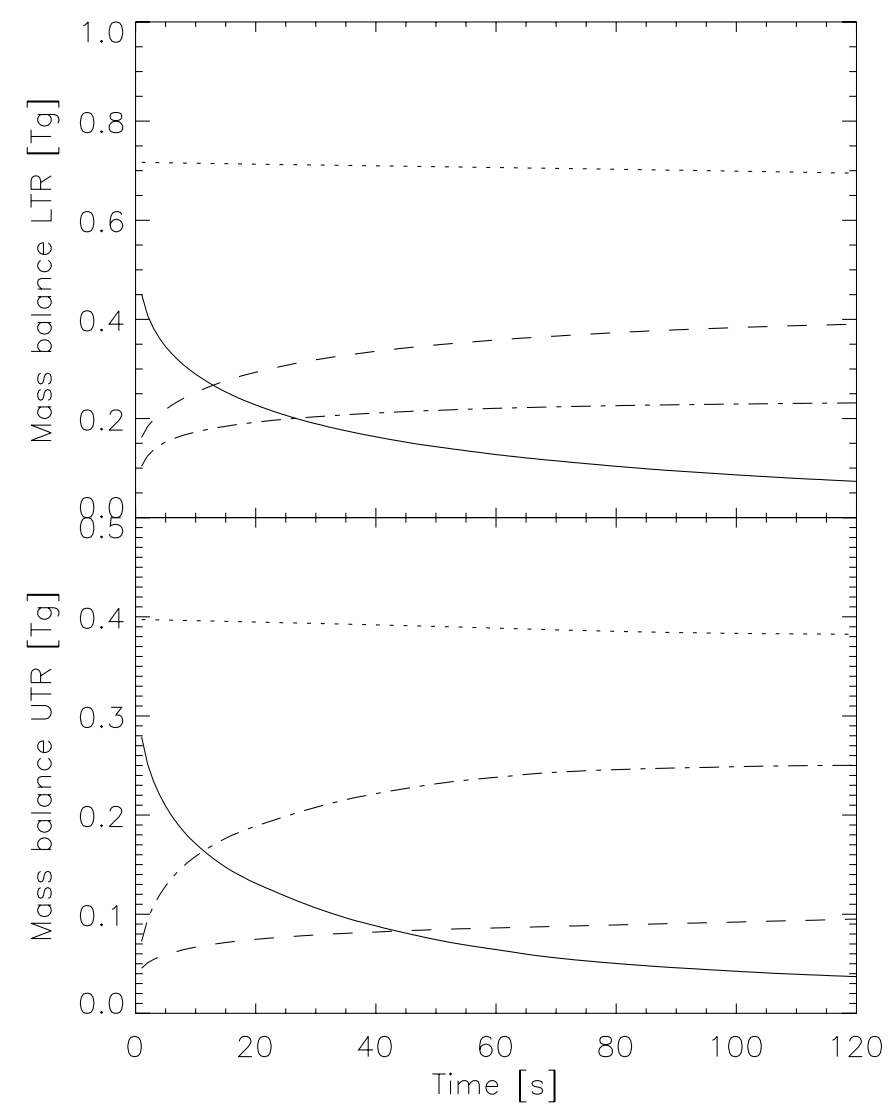

Figure 6. Mass budget of the transition region (tracer fluid): material remaining in the original temperature range $\left(\left[2.0 \times 10^{4}, 3.0 \times 10^{5}\right] \mathrm{K}\right.$ top panel, $[3.0 \times$ $10^{5}, 7.0 \times 10^{5}$ ] K bottom panel) is shown by the solid line. The dashed line shows material cooling to temperatures below the original lower temperature, and the dash-dotted line shows material heated to temperatures above the original range. The dotted line shows the total mass in the initial temperature ranges over 2 minutes (not the traced material).

as the amount of traced material that remains in the originally defined temperature range (solid line). Keeping in mind that the total amount of mass in the transition region is nearly constant during the entire one hour run of simulation B1, it is clear that the most striking feature of the figure is the extreme rapidity which the tracer material leaves the original temperature range: in both upper and lower transition regions we find that only $10 \%$ of the original fluid remains after $120 \mathrm{~s}$. Mass is cycled between the various atmospheric regions extremely rapidly in this simulation.

This is mainly due to the large amplitudes of the physical processes that dominate transition region dynamics and energetics: mass flows and rapid episodic heating (to a small extent it is also due to the diffusive nature of the Bifrost code combined with the large initial density gradients at the edges of the tracer material). The lower transition region material is mainly lost to the chromosphere, but there is a significant fraction of material that is heated to temperatures greater than $3 \times 10^{5} \mathrm{~K}$. In the upper transition region the opposite is true: most material removed from this temperature range is heated to greater temperatures, with a smaller fraction cooling to lower transition region and chromospheric temperatures. Also plotted, as the dotted line, in Figure 6 is the amount of material (not the tracer fluid) that lies in the defined temperature range as a function of time. As noted earlier in the introduction, both the lower and upper transition region are found to maintain a roughly constant mass during the tracer fluid simulation, as indeed they do during the entire

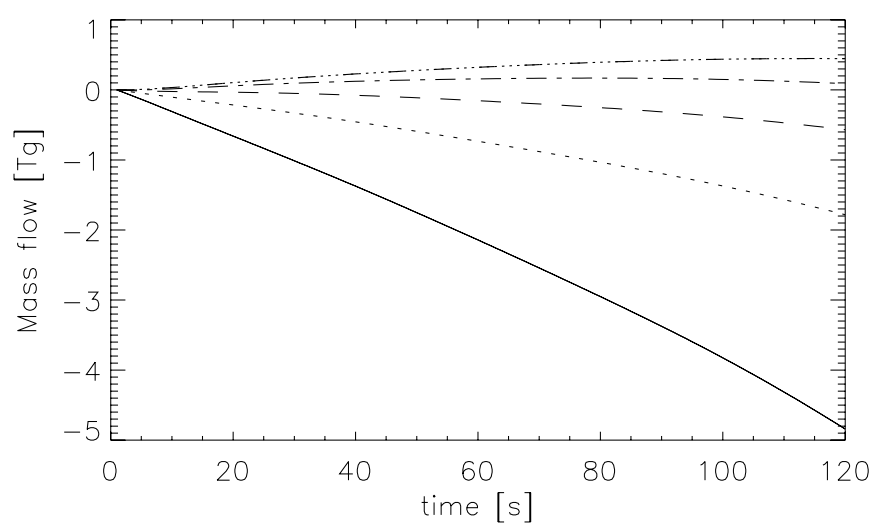

Figure 7. Mass flows in the transition region integrated in time to show the net movement of mass at different temperatures: $T=2 \times 10^{4} \mathrm{~K}$ (solid), $10^{5} \mathrm{~K}$ (dotted), $3 \times 10^{5} \mathrm{~K}$ (dashed), $5 \times 10^{5} \mathrm{~K}$ (dot-dashed), and $7 \times 10^{5} \mathrm{~K}$ (tripledot-dashed). Negative values signify downward motions.

$1 \mathrm{hr}$ period of the B1 simulation. Thus, even though the processes that move mass from one temperature range to another are very rapid there exists a balance between them such that the amount of material at transition region temperatures is more or less constant.

Let us first consider the role of mass flows. In Figure 7 we show the total mass transported by mass motions at various temperatures in the transition region between $2 \times 10^{4} \mathrm{~K}$ and $7 \times 10^{5} \mathrm{~K}$. Essentially, we integrate the mass flux over the surface of the specified temperatures. In a steady state flow situation one would expect an equal amount of mass to cross each temperature surface. Figure 7 shows that this is not the case: a very large amount of material cools and exits the transition region through the isothermal layer defined by $2 \times 10^{4} \mathrm{~K}$. Unless compensated, the amplitude of this flow is enough to empty the transition region of all material in less than a minute. The flow of material flowing into the lower transition region down through the layer at $3 \times 10^{5} \mathrm{~K}$ falls short by some $3 \mathrm{Tg}$ in refilling the lower transition region. The situation is the same for higher temperatures, indeed, in the upper transition region at temperatures above $5 \times 10^{5} \mathrm{~K}$ we see that material is rising on average, supplying the hotter corona above with new material. Thus, there is no steady state flow from coronal to chromospheric temperatures, nor the opposite from chromospheric to coronal.

Since we have measured the lower transition region mass to be more or less constant in time we can construct an absolute mass budget for the transition region by looking at the amounts of heated and cooled material coming from the tracer fluid and scaling these to the amount of material remaining in the tracer fluid, assuming that the fractional losses remain constant in time. We therefore normalize the amount of tracer material lost from the lower transition region, $\left[2.0 \times 10^{4}, 3.0 \times 10^{5}\right] \mathrm{K}$, and from the upper transition region, $\left[3.0 \times 10^{5}, 7.0 \times 10^{5}\right] \mathrm{K}$, by multiplying the material lost at a given time by the ratio of the original mass to the mass remaining in the original temperature ranges $\left[m_{\mathrm{TR}}(t=0) / m_{\mathrm{TR}}(t)\right]$. Let us first look at material cooling from the lower transition region. Figure 8 shows that the amount of cooling tracer material coming from the lower transition region temperatures is nearly the same as that measured in Figure 7 to flow down at $2 \times 10^{4} \mathrm{~K}$, i.e., some 4-5 Tg during the model run. Thus, most of the mass loss from the bottom of the transition region is in the form of flows to cooler temperatures. The amount of material cooling from the upper transition region to temperatures below $3 \times 10^{5} \mathrm{~K}$ is 


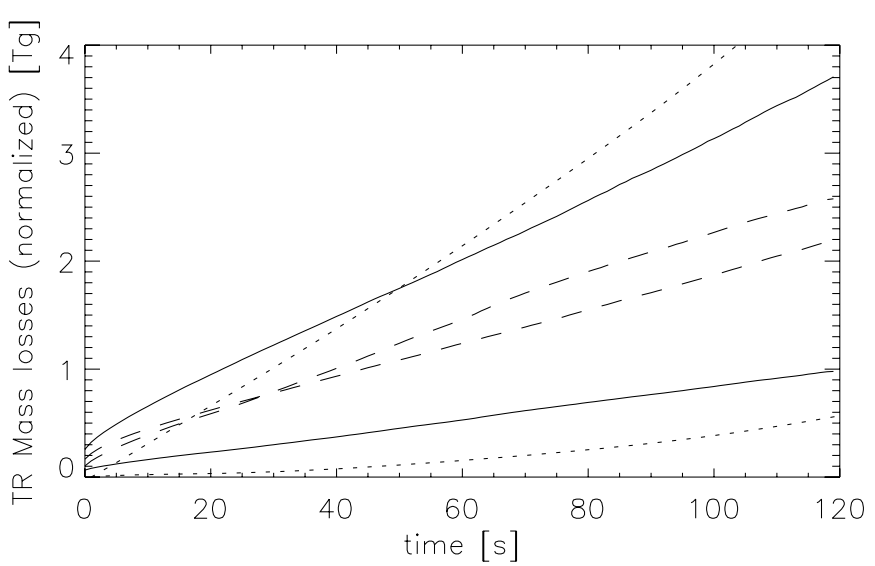

Figure 8. Mass losses to cooler regions from the lower transition region (upper solid line) and from the upper transition region (lower solid line) and to hotter regions from the lower and upper transition region (dashed lines). These curves are normalized by multiplying the mass lost from the original temperature range by the ratio of the original mass to the mass remaining in the original temperature range $\left[m_{\mathrm{TR}}(t=0) / m_{\mathrm{TR}}(t)\right]$. For reference, the mass flow loss rates from the bottom of the lower and upper transition region are overplotted (dotted lines; the upper dotted line is mass flow from the bottom of the lower transition region).

seen to be about $1 \mathrm{Tg}$, some of which is in the form of flows $(0.5 \mathrm{Tg})$ as attested by Figure 7 . The dashed lines in Figure 8 shows the (scaled) amount of material heated out of the lower and upper transition region. The lower transition region loses a significant fraction of its mass as a result of material heating out of the temperature band, some $2 \mathrm{Tg}$ during the period of the tracer fluid calculation. An approximately equal amount of material is heated to coronal temperatures from the upper transition region. Figure 7 shows a certain amount of material that flows to temperatures greater than $7 \times 10^{5} \mathrm{~K}$ through the top of the upper transition region, some $0.5 \mathrm{Tg}$ in $120 \mathrm{~s}$. However, this is still much less than the estimate shown in Figure 8 of roughly $2 \mathrm{Tg}$ heated to temperatures above $7 \times 10^{5} \mathrm{~K}$; much of this heating must occur in place and not be associated with any material flow.

To summarize the global mass balance through the transition region and into the corona: a large amount of material continually cools to chromospheric temperatures from the lower transition region, $\left[2 \times 10^{4}, 3 \times 10^{5}\right] \mathrm{K}$. The cooling plasma is largely in the form of flows with an average downward velocity of $3-4 \mathrm{~km} \mathrm{~s}^{-1}$ at $2 \times 10^{4} \mathrm{~K}$. Figures 7 and 8 show that this large mass loss is not replaced by material flowing down from the upper transition region, nor from upper transition material cooling in place. Instead, upper chromospheric material must continually be heated in place, at least to lower transition region temperatures, on average replacing the matter that is lost through the large average downflow. Note that most of this material is only heated to temperatures below $3 \times 10^{5} \mathrm{~K}$; only a fraction is brought to upper transition region and coronal temperatures.

Most of the mass lost from the upper transition region, defined here as plasma in the range $\left[3 \times 10^{5}, 7 \times 10^{5}\right] \mathrm{K}$, is heated or flows to coronal temperatures. A significant fraction is also lost as downflow and/or through cooling to lower temperatures. As in the upper chromosphere, significant amounts of both lower and upper transition region material is heated in place; in the upper transition region there is also some flow of material to greater temperatures.

Mass exchange in the transition region and between the transition region and upper chromosphere is quite rapid, with total mass replacement timescales of the order of minutes or less. The coronal mass balance has a somewhat longer timescale. With this in mind, let us now study the properties of the tracer plasma in greater detail. Figure 9 shows the evolution of the temperature of the material originally in the lower transition region. The left panel shows the minimum, maximum, and mass weighted average temperature or the expected value of the temperature in the traced mass distribution, $\langle\psi T\rangle /\langle\psi\rangle$, while the right panel shows the distribution of temperature at four separate times: $t=0,10,30,120 \mathrm{~s}$. Initially the average tracer temperature is about $7.5 \times 10^{4} \mathrm{~K}$. This increases rapidly, within a few seconds, to $1.6 \times 10^{5} \mathrm{~K}$ at $t=5 \mathrm{~s}$. The average temperature continues to increase after this, but somewhat more slowly, reaching a maximum of $2.5 \times 10^{5} \mathrm{~K}$ at about $t=55 \mathrm{~s}$. After this time the average temperature of the tracer fluid is more or less constant. A small percentage of the plasma is heated to great temperatures very rapidly and the maximum temperature jumps from $3.0 \times 10^{5} \mathrm{~K}$ to $1.2 \times 10^{6} \mathrm{~K}$ already in the first second. Thereafter, the maximum temperature increases little, at $t=120 \mathrm{~s}$, we find it to be approximately $2.0 \times 10^{6} \mathrm{~K}$. The minimum temperature also falls very rapidly, decreasing from $2.0 \times 10^{4} \mathrm{~K}$ to about $5.0 \times 10^{3} \mathrm{~K}$ in the first second. The minimum temperature continues to decrease until the end of the run where we find the minimum temperature of the tracked plasma to be $3.5 \times 10^{3} \mathrm{~K}$.

The right panel shows that the distribution of temperature rapidly splits into two separate populations, one of which is centered around $10^{4} \mathrm{~K}$, while the other contains hot plasma with temperatures above $10^{6} \mathrm{~K}$. These distributions are made by considering the amount of material (mass fraction $\Delta m / m_{\mathrm{T}}$ of the total tracer mass) at a given temperature as a function of time. The temperature bin size is set to $\Delta \lg (T)=0.1$ and the fraction of tracked material, $\Delta m$, in each temperature interval, $\Delta \lg (T)$, normalized with the total mass in the initial volume, $m_{\mathrm{T}}$. Since the pressure is nearly constant in the transition region along any given field line, the amount of material is much greater at lower temperatures: initially, $25 \%$ of the tracer mass is in the temperature range $\left[2.0 \times 10^{4}, 2.5 \times 10^{4}\right] \mathrm{K}$, $50 \%$ between $\left[2.5 \times 10^{4}, 1.0 \times 10^{5}\right] \mathrm{K}$, and $25 \%$ in the range $\left[1.0 \times 10^{5}, 3.0 \times 10^{5}\right] \mathrm{K}$. Already after $10 \mathrm{~s}$ we see significant changes to this distribution: a large fraction of the tracer material is cooling to chromospheric temperatures. In addition, a significant but smaller fraction of the material is being heated. We find that at $t=30 \mathrm{~s}$, some $20 \%$ has been heated to temperatures above $3.0 \times 10^{5} \mathrm{~K}$. As noted above, very little material remains in the original temperature range after $120 \mathrm{~s}$.

The evolution of transition region plasma heights are shown in Figure 10. The left panel shows that the average height is very nearly constant over the $120 \mathrm{~s}$ simulation, initially at $1.65 \mathrm{Mm}$, and ending some $100 \mathrm{~km}$ lower, at $1.56 \mathrm{Mm}$. The maximum height, initially at $3.3 \mathrm{Mm}$, increases substantially to $3.8 \mathrm{Mm}$ in the first few seconds, thereafter rising in fits and starts to 7.4 $\mathrm{Mm}$ by the end of the run. (The stair-like behavior of the maximum height plot is due to the uneven distribution of the grid points for the $z$ axis.) The minimum height of the tracer material is initially at $1.2 \mathrm{Mm}$ and drops smoothly, to the mid chromosphere, $0.6 \mathrm{Mm}$ at $t=120 \mathrm{~s}$.

The large maximum height found above shows that some of the material is propelled to great heights, but the right panel of Figure 10 shows that the actual fraction of mass that effectively moves upward is in fact small compared with the total mass tracked. This is a simple consequence of the fact that the atmosphere is exponentially stratified and that there 

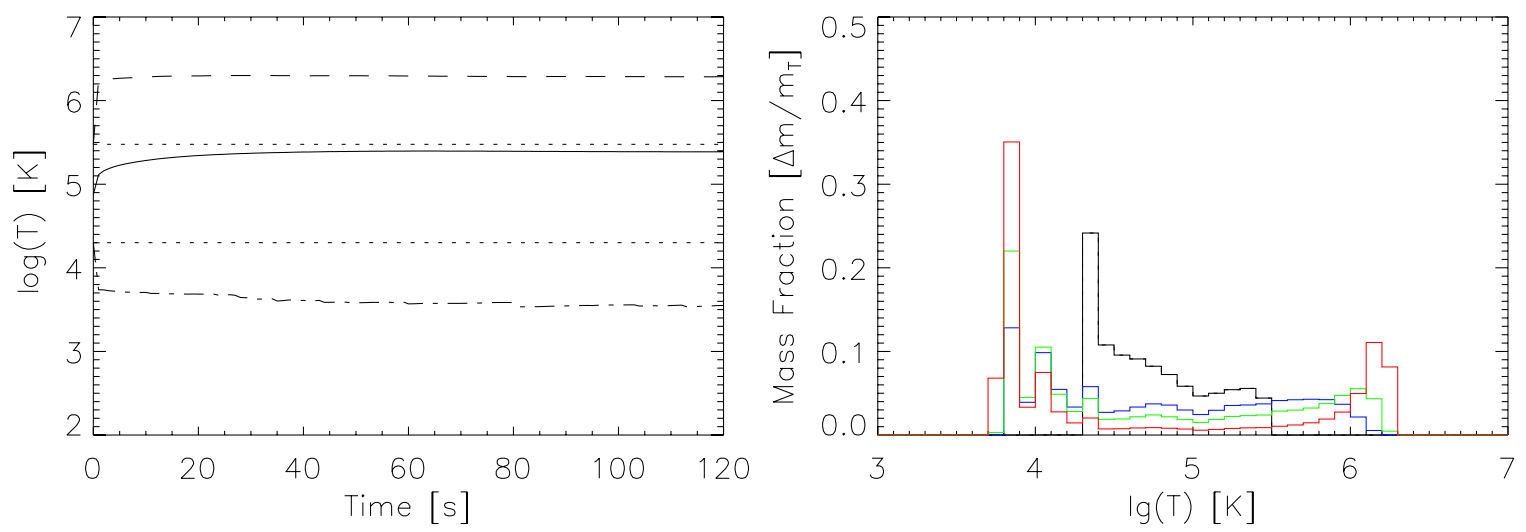

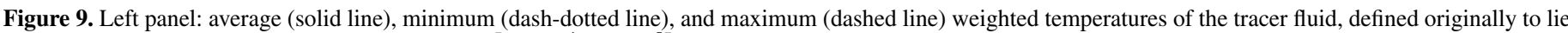

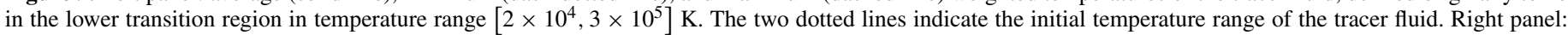

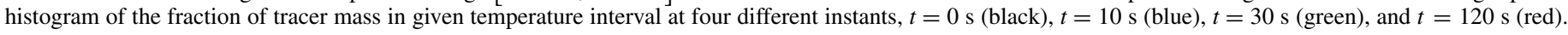
The bin sizes are $\Delta \lg (\mathrm{T})=0.1$.
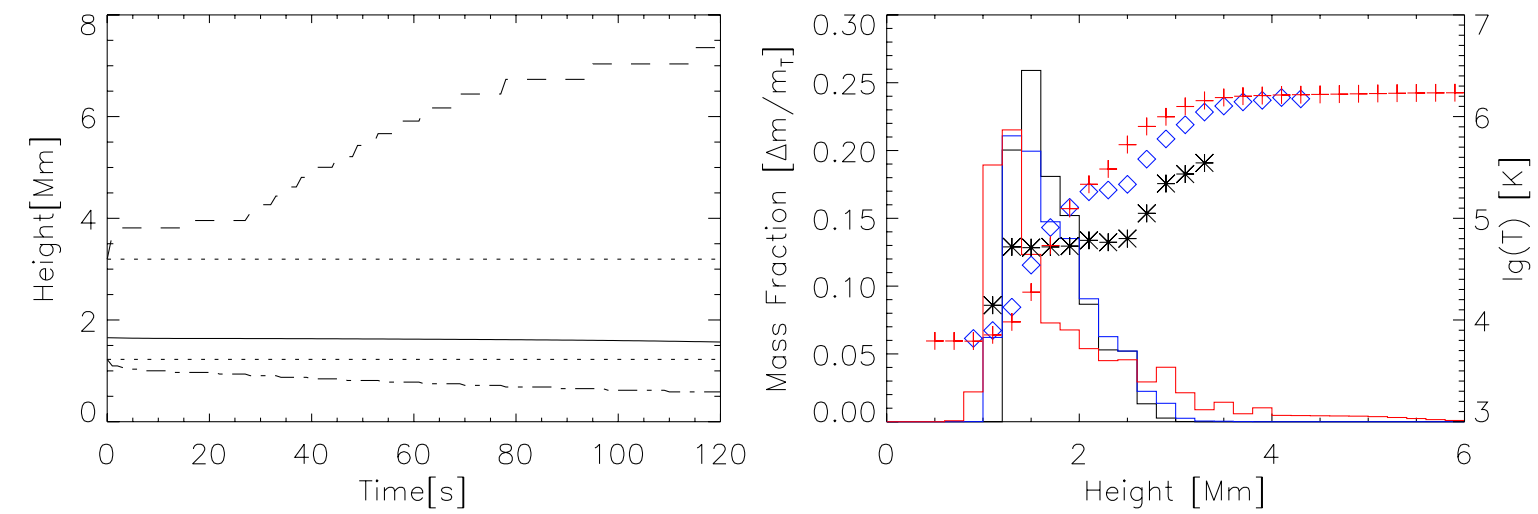

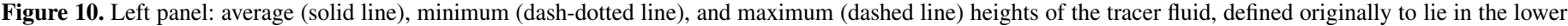

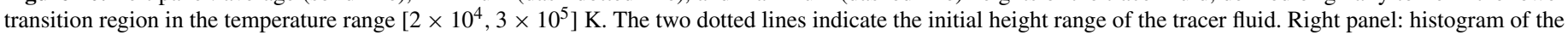

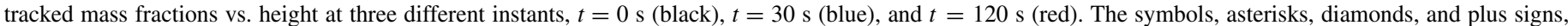
correspond to the average temperature of the material in each bin. The bin sizes are $0.2 \mathrm{Mm}$.

therefore is much more chromospheric than coronal material. This panel shows the histograms of the tracked mass fractions versus height at three different instants, $t=[0,30,120] \mathrm{s}$, and the size of the height bin is $\Delta h=0.2 \mathrm{Mm}$. Little change in these histograms is evident before $120 \mathrm{~s}$ has passed. However, at this time we find that about $25 \%$ of the material has moved below the original $1.2 \mathrm{Mm}$ height, while as much as $5 \%$ of the tracked material has moved above $3.0 \mathrm{Mm}$ and there is a significant tail of material reaching up to heights of $6 \mathrm{Mm}$. Thus a large fraction of the tracer material remains in the original height band $[1.2,3.0] \mathrm{Mm}$ even though the temperature of almost all the material has changed significantly. Perhaps not surprisingly, the average temperature of the material that has moved significantly upward has increased to above $10^{6} \mathrm{~K}$ while the average temperature of the material found at low heights decreases.

Figure 11 shows the distribution of velocities versus the distribution of temperatures at the same times as in the previous figures. The top right panel at $t=10 \mathrm{~s}$ and bottom left panel at $t=30 \mathrm{~s}$ show that populations of material with temperatures $<2 \times 10^{4} \mathrm{~K}$ and with $>3 \times 10^{5} \mathrm{~K}$ are quickly established. There is a tail of hot, high upward velocity material, but there is also a significant fraction of material heated to coronal temperatures that is seen to have small velocities or indeed move downward.

Finally, since plasma motions and thermal conduction are constrained to flow along the magnetic field in the low $\beta$ corona we look at loop structures to better understand the mass flows.

As a general rule, we find two types of behavior when looking at closed loops; these types differ according to the maximum height of the loop apex. Loops with apex heights $z<2.5 \mathrm{Mm}$ or so are vigorously heated but rarely attain temperatures above $1.0 \times 10^{6} \mathrm{~K}$. Material in these loops is rapidly cycled in temperature and density. On the other hand, higher loops with tops above, say, $4 \mathrm{Mm}$ will largely retain coronal temperatures for periods much longer than the typical recurrence rate of dissipation events. This difference is mainly due to the strong density stratification of the upper chromosphere and lower corona: high density low loops are associated with much greater radiative losses since these scale as $\rho^{2}$ and low loops therefore cool much more rapidly despite the vigorous heating.

We show two examples of the first kind of loop in Figures 12 and 13 . In both cases we trace the field line location, heating rate, temperature, particle density, and velocity along the magnetic field for a $240 \mathrm{~s}$ period. In Figure 12 a short loop, with a length of $6 \mathrm{Mm}$ as measured from photosphere to photosphere and apex height of $2 \mathrm{Mm}$ is rapidly heated from $T=3.0 \times 10^{4} \mathrm{~K}$ to $T=3.0 \times 10^{5} \mathrm{~K}$ in the $240 \mathrm{~s}$ covered as the heating rate per particle increases from $0.01 \mathrm{GW} \mathrm{kg}^{-1}$ to $0.1 \mathrm{GW} \mathrm{kg}^{-1}$. The increased temperature leads to an overpressure in the vicinity of the loop apex and material is pushed out of the hotter part of the 

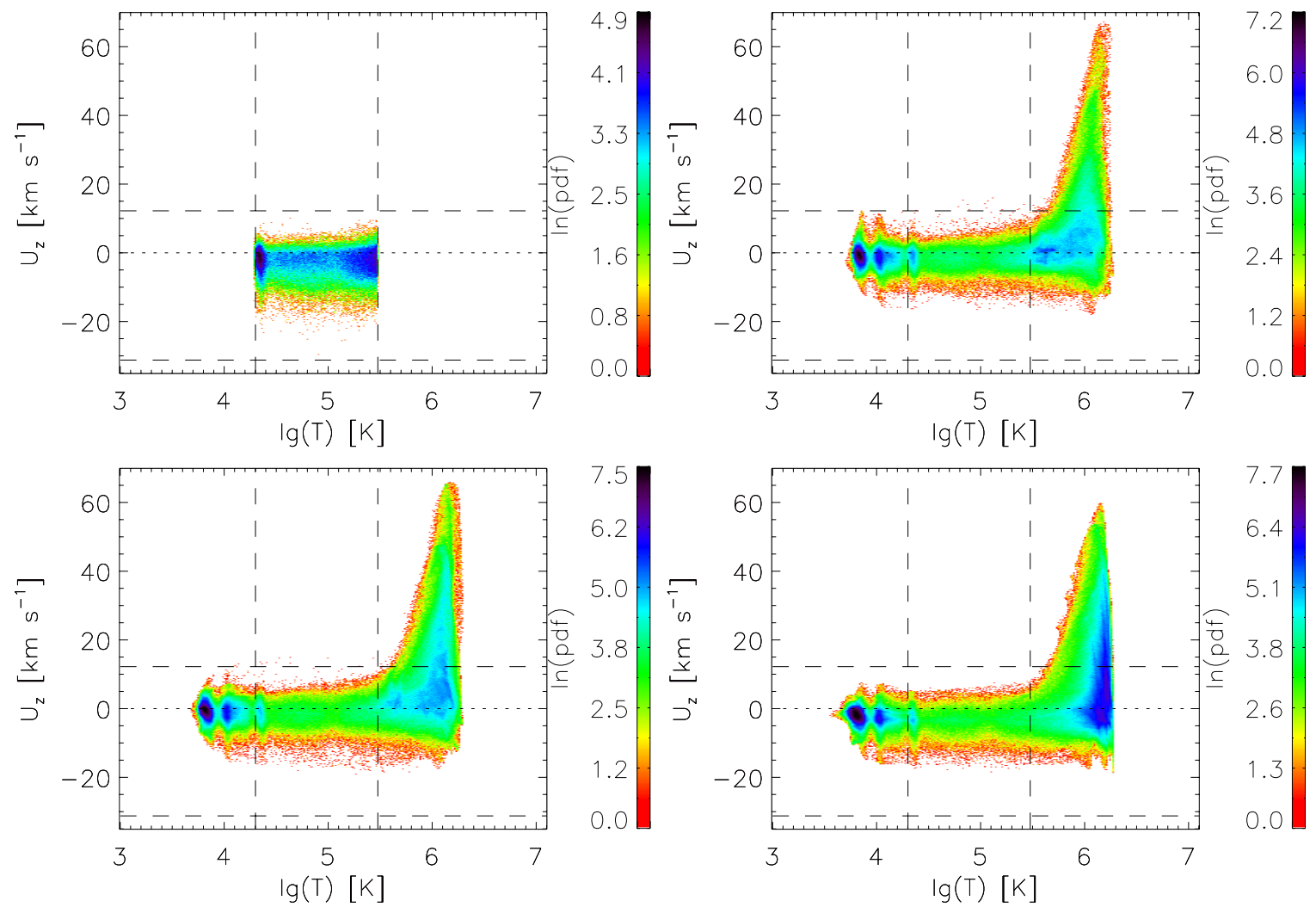

Figure 11. Probability density function of the velocity vs. lg temperature at four different instants, $t=0 \mathrm{~s}$ (top left panel), $t=10 \mathrm{~s}$ (top right panel), $t=30 \mathrm{~s}$ (bottom left panel), and $t=120 \mathrm{~s}$ (bottom right panel). The black region corresponds to a high density of cells, and red regions correspond to a small density of cells. The dashed lines show the initial cutoff for the variables in the initial model.
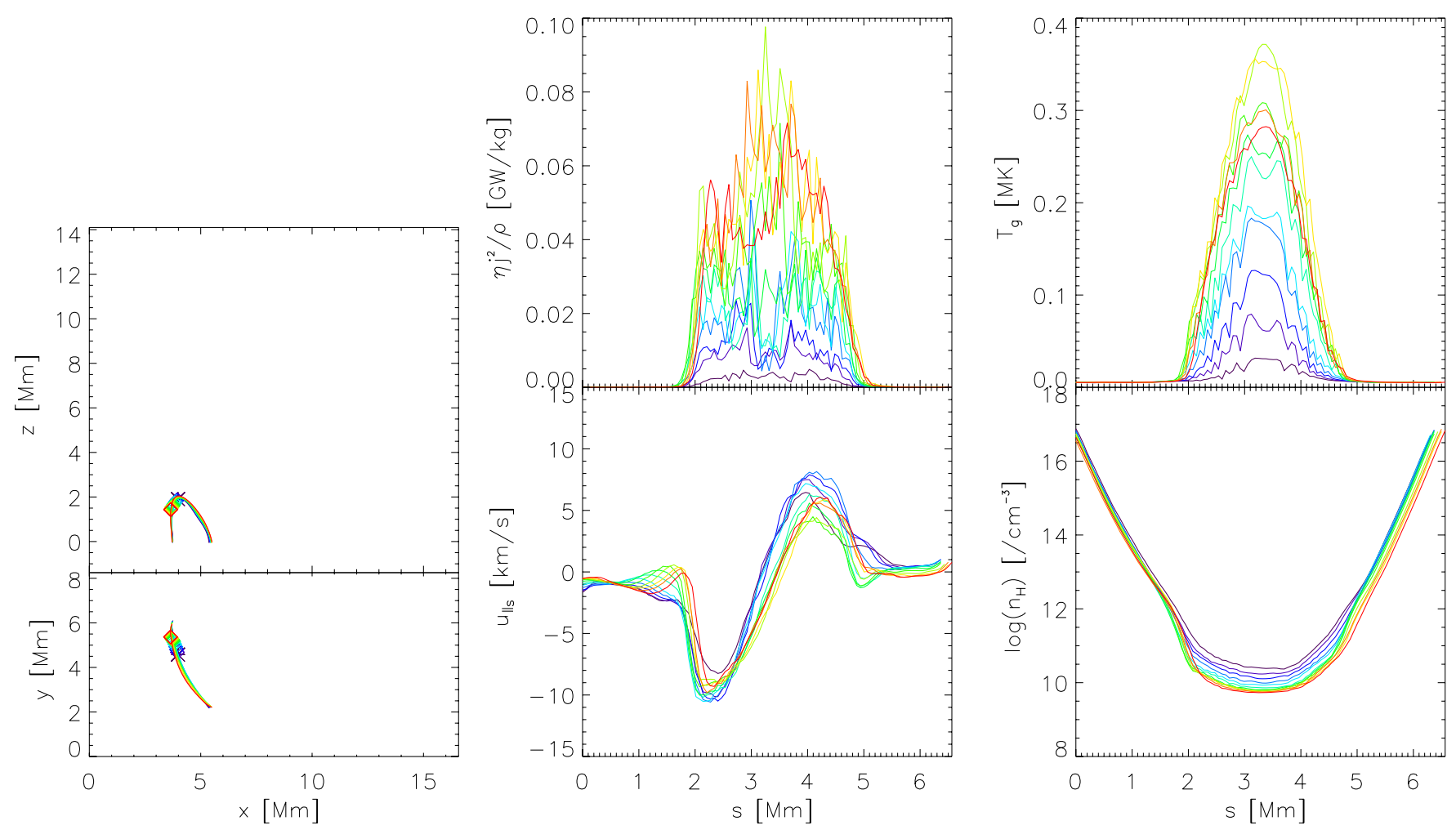

Figure 12. Magnetic field lines projected on $x-z$ and $x-y$ planes (left panels), specific heating rate $\eta j^{2} / \rho$ and velocity along the field line $s$ (middle panels), and temperatures and hydrogen particle densities along the field line (right panels). In this figure we follow a relatively low, short loop that is being heated over a $240 \mathrm{~s}$ period at $20 \mathrm{~s}$ intervals - from black at $0 \mathrm{~s}$, via blue and turquoise, to yellow and red when $t=240 \mathrm{~s}$ at the end of the time series shown. Velocities along the loop are positive in the direction of increasing loop coordinate $s$. The symbols in the left panel represent a starting point of a comoving sequence of the reference point for the integrated field line. 

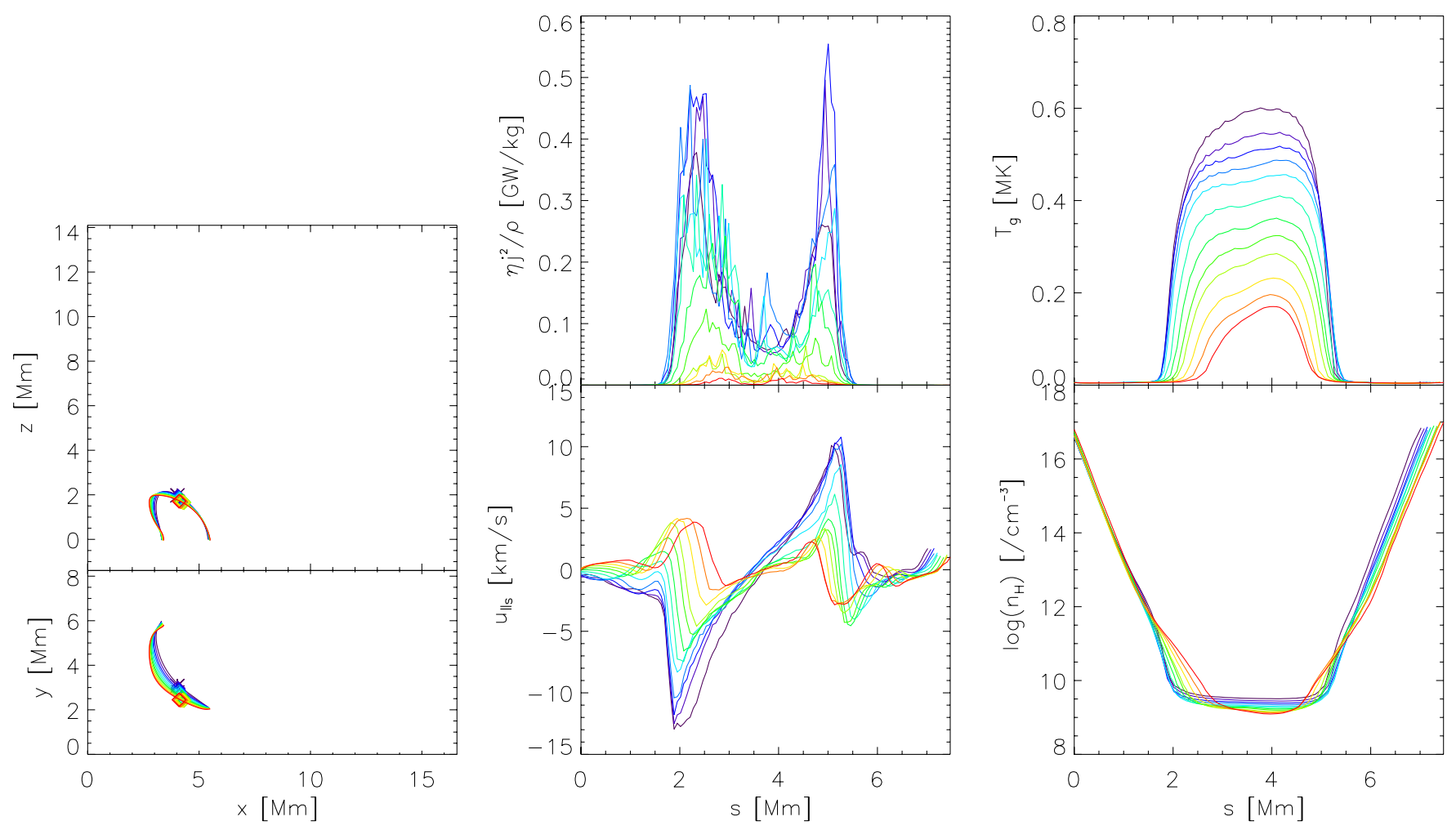

Figure 13. As Figure 12 but for a relatively low, short loop that is cooling.

loop at velocities of up to $10 \mathrm{~km} \mathrm{~s}^{-1}$ (the velocity plotted is the velocity along the magnetic field, thus a downflow is negative for $s<0.5 \times$ loop length and positive thereafter).

Note that for this short loop the particle density, and indeed the amount of material in the upper region of the loop, decreases from $4 \times 10^{10} \mathrm{~cm}^{-3}$ initially to $5 \times 10^{9} \mathrm{~cm}^{-3}$ as the loop is heated.

A short, cooling, loop is shown in Figure 13. In this case the heating rate per particle is originally concentrated near the loop apex at some $0.5 \mathrm{GW} \mathrm{kg}^{-1}$ and the apex temperature is $6.0 \times 10^{5} \mathrm{~K}$. Recently heated material is still being pushed out of the loop at velocities of $13 \mathrm{~km} \mathrm{~s}^{-1}$ in one loop leg and $10 \mathrm{~km} \mathrm{~s}^{-1}$ in the other. As time progresses, the heating rate initially remains high or increases in the upper chromosphere foot points, but rapidly decreases to a maximum of $0.01 \mathrm{GW} \mathrm{kg}^{-1}$ at the end of the period covered. The loop temperature falls rapidly to less than $1.5 \times 10^{5} \mathrm{~K}$ and the lower pressure at the loop apex pulls material into the loop at a velocity of up to $5 \mathrm{~km} \mathrm{~s}^{-1}$ in both legs. The apex density is of order $10^{10} \mathrm{~cm}^{-3}$ throughout the period covered, but the legs of the loop become denser as the loop cools.

An example of the typical behavior of longer loop is shown in Figure 14. In this case we follow a high loop of length $30 \mathrm{Mm}$, from photosphere to photosphere, with an apex height of some $11 \mathrm{Mm}$, for $240 \mathrm{~s}$. This loop is variably and weakly heated, with the heating concentrated near both footpoints during the entire period. The temperature structure in the loop does not change much with time, but the coronal part of the loop extends by a few hundred kilometers as heated material is brought into the loop as a result of chromospheric evaporation. The loop apex temperature is roughly $1.3 \times 10^{6} \mathrm{~K}$. The velocity pattern in the loop is also typical of longer loops with strong upflows of some $10-12 \mathrm{~km} \mathrm{~s}^{-1}$ and $15-20 \mathrm{~km} \mathrm{~s}^{-1} \mathrm{~s}$ in the footpoints just above the most strongly heated plasma. In the part of the footpoints just below the strongly heated regions, on the other hand, we find material being pushed downward toward the chromosphere at velocities of roughly $5 \mathrm{~km} \mathrm{~s}^{-1}$.

\section{DISCUSSION AND CONCLUSION}

In this paper we try to shed some light on the mass balance and mass transport in the upper solar atmosphere. In particular we focus on the mass cycling in the upper chromosphere, transition region, and corona. Our simulations show that the coronal mass is kept roughly constant or even increases despite the pervading down flow in the upper chromosphere and lower transition region, as shown observationally by Peter \& Judge (1999) and simulated by Hansteen et al. (2010).

The introduction of the tracer fluid in the lower transition region, $\left[2.0 \times 10^{4}, 3.0 \times 10^{5}\right] \mathrm{K}$, at a certain point in time shows that material is found inside this temperature range for a short period of time. After two minutes solar time about, $95 \%$ of the material had left this region, about $70 \%$ of which has cooled to chromospheric temperatures, while about $25 \%$ of the material is heated to greater, upper transition region or coronal, temperatures. The remaining $5 \%$ of the material remains in the initial temperature range.

Heating and cooling are accompanied by mass motions. The results show that for material originally in the temperature range $\left[2.0 \times 10^{4}, 3.0 \times 10^{5}\right] \mathrm{K}$ heated material largely flows upward. Some small fraction of this at high velocity, of order $50 \mathrm{~km} \mathrm{~s}^{-1}$, but mostly at lower velocities, $10 \mathrm{~km} \mathrm{~s}^{-1}$ or less. There is also a significant fraction of heated material that is propelled downward at velocities of some $-5 \mathrm{~km} \mathrm{~s}^{-1}$. Material cooling to temperatures lower than $2.0 \times 10^{4} \mathrm{~K}$ is mainly found to be downflowing with velocities between 2 and $4 \mathrm{~km} \mathrm{~s}^{-1}$. On average, heated material flows upward, while cooling material flows down. Despite the large velocities found in the up flowing material only a small fraction, $10 \%$, is being displaced higher than the original highest location $(3.3 \mathrm{Mm})$ of the tracer fluid. 

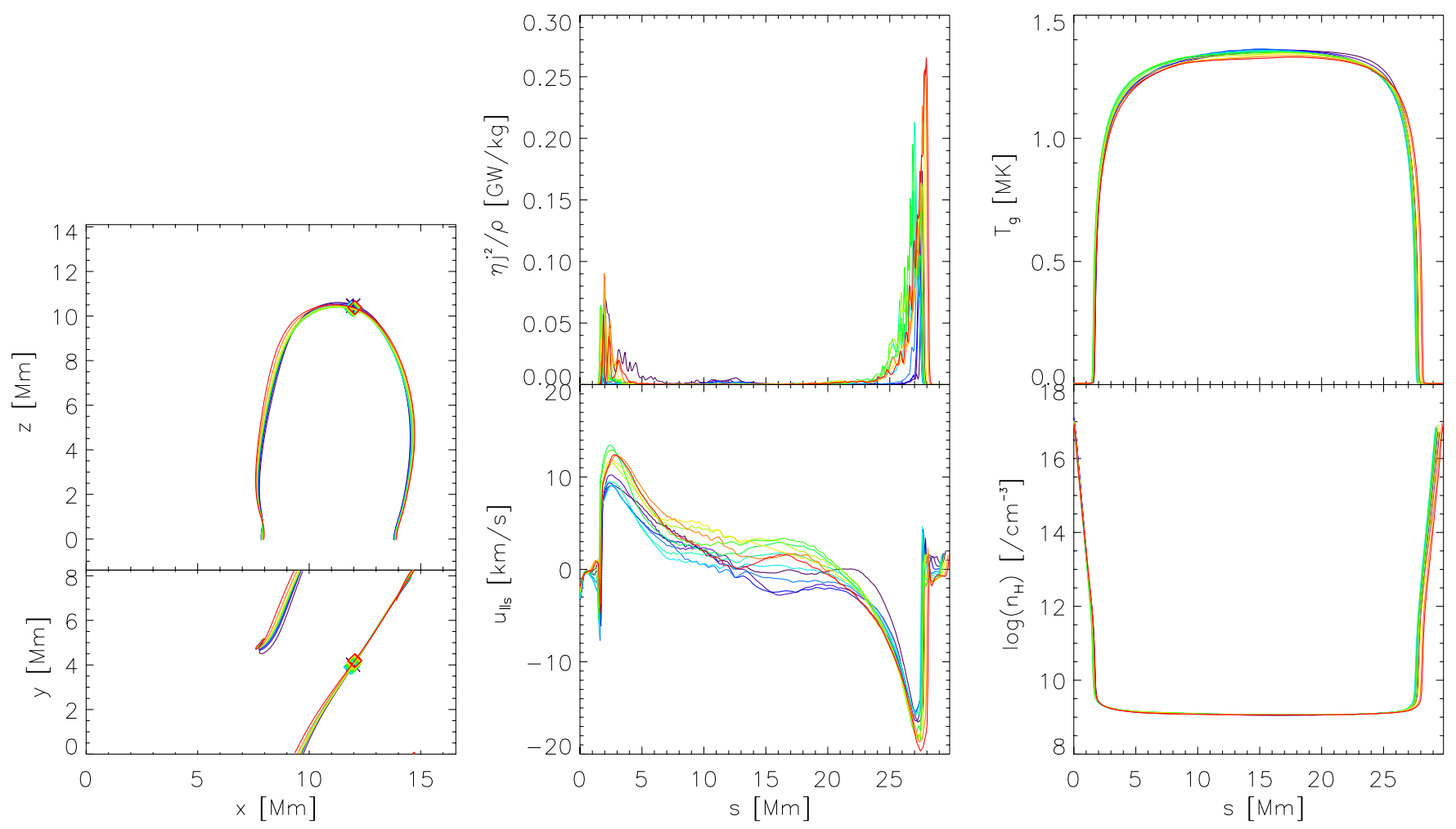

Figure 14. As Figure 12 but for a high coronal loop that is only moderately heated.

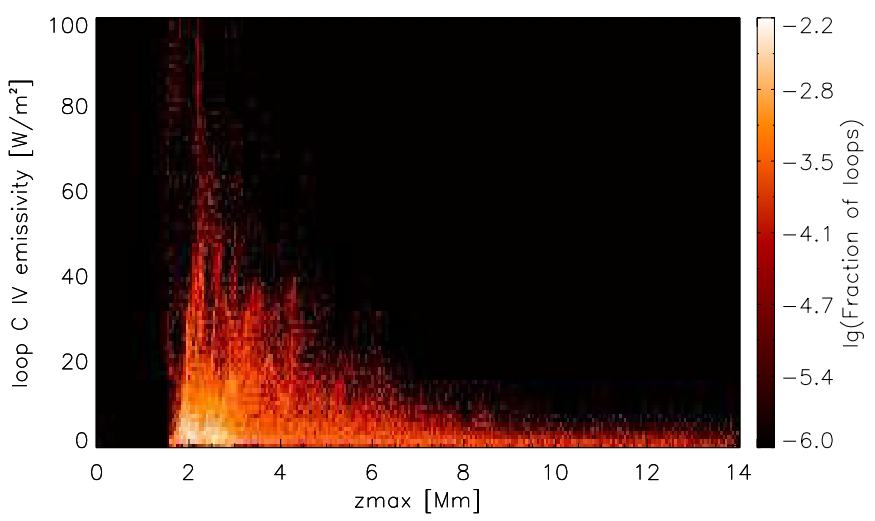

Figure 15. Fraction of loops with temperatures $10^{5} \mathrm{~K}$ or greater attaining height $z_{\max }$. The histogram also shows the distribution of the total emissivity of these loops in the typical lower transition region spectral line C IV $1548 \AA$ A: most transition region loops are low lying and low lying loops are the most strongly emitting in the transition region lines.

We conclude that most of the material seen in transition region lines is heated rapidly from chromospheric to lower transition region temperature $<3.0 \times{ }^{5} \mathrm{~K}$ and is thereafter "pushed down" as it cools. The latter is due to the increased pressure gradient force resulting from the heated plasma. Since we mainly find down flows, this implies that the bulk of the transition region material resides in small loops, with the loop tops located some $2 \mathrm{Mm}$ above the photosphere, two examples of which are shown in the previous section.

Support for this can be found by considering all the loops that reach temperatures of at least $10^{5} \mathrm{~K}$. We find the maximum height of all such loops by integrating along the magnetic field lines. As can be seen in Figure 15 the vast majority of loops reach heights of only $<4 \mathrm{Mm}$. This is of course not surprising since the total signed magnetic field in the simulation is small, such that most field lines connect regions not too far apart in the photosphere. On the other hand, this is likely to be fairly representative of the magnetic "salt and pepper" conditions found in the quiet Sun. In addition, Figure 15 also shows that these low lying loops stand for the major portion of transition region emission, as illustrated by the distribution of the total emissivity for the C IV $1548 \AA$ line calculated along each loop from the standard CHIANTI $g\left(T, n_{\mathrm{e}}\right)$ function (Dere et al. 2009).

In these low lying loops the density is high and radiative cooling is efficient; as material cools to chromospheric temperatures the overpressure in the upper part of the loop vanishes and cool, $<2.0 \times 10^{4} \mathrm{~K}$, material is free to flow back up into the loops.

In longer loops that extend to coronal heights $>3 \mathrm{Mm}$ or so above the photosphere, heated material can rise rapidly along nearly vertical field lines and the lower density material found at greater heights is more easily heated to coronal temperatures of $1 \mathrm{MK}$ or more. Thus, material initially at temperatures $>5.0 \times 10^{5} \mathrm{~K}$ or so on average flows upward. This material will remain at elevated temperatures longer than that found in short loops, since thermal conduction and radiative losses at low density are relatively slow processes compared to radiative losses at the higher densities found in short loops. Cooling material at higher temperatures remains mainly in place.

These results are typical of the simulations performed as the results found in other tracer fluid calculations, starting at $t=30, t=50, t=55$ minutes are consistent with the scenario presented here. The bulk of lower transition region material at those times is heated to temperatures below $3.0 \times 10^{5} \mathrm{~K}$, thereafter to be pushed downward while cooling. These short cool loops may bear some relation to the "unresolved fine structure" reported by Feldman et al. (1999) and in references cited therein. Some percentage of heated material, mainly that found along field lines nearly vertical in the region of 
greatest heating per particle $[1.5,3] \mathrm{Mm}$, is raised to coronal temperatures and is free to expand to greater heights. While we find some small variations in the amount of material heated to coronal temperatures, on average we find that about $10 \%$ of all material is effectively displaced upward and to coronal temperatures in all the experiments performed. The dichotomy between low cool loops and hot coronal loops is in part due to the fact that in this model, most loops are relatively short and hence dense, and that radiative cooling is most efficient at lower transition region temperatures.

To summarize the findings of this paper: most transition region material and emission are found in short loops such as those shown in Figures 12 and 13. These low lying loops are dense, and even when heated vigorously seldom achieve temperatures above $3 \times 10^{5} \mathrm{~K}$. When heated, they show large redshifts as the increased pressure pushes material out of the loop. When cooling, the pressure falls near the loop top and the loops are refilled with mass at low $\left(T<2 \times 10^{4} \mathrm{~K}\right)$ temperature. However, since these loops are cool, their diagnostics are largely irrelevant when considering the coronal mass balance. Higher loops, even when heated near the footpoints, are much more easily raised to coronal temperatures and therefore contain the bulk of coronal material. Their mass balance is controlled by chromospheric evaporation as hinted at in Figure 1, exemplified in Figure 14, and as explained in many papers previously (Klimchuk 2006; Reale 2010). Based on these arguments, we could therefore expect that the ratio of cool to hot loops will vary with the larger scale structure of the magnetic field and that we therefore will find the transition region to have varying global properties (e.g., different average red shift) in regions such as coronal holes or in plage where the large scale magnetic field structure presumably is different than in the quiet Sun.

This research was supported by the European Commission funded Research Training Network SOLAIRE. It was also supported by the Research Council of Norway through grants of computing time from the Norwegian Programme for Supercomputing. We thank M. Carlsson for reading and commenting on the paper.

\section{REFERENCES}

Athay, R. G. 1984, ApJ, 287, 412

Athay, R. G., \& Holzer, T. E. 1982, ApJ, 255, 743

Athay, R. G., \& White, O. R. 1978, ApJ, 226, 1135

Bingert, S., \& Peter, H. 2011, A\&A, 530, A112

Bradshaw, S. J., \& Cargill, P. J. 2010, ApJL, 710, L39

Brekke, P., Hassler, D. M., \& Wilhelm, K. 1997, SoPh, 175, 349

Carlsson, M., \& Leenaarts, J. 2012, A\&A, 539, A39

Chae, J., Yun, H. S., \& Poland, A. I. 1998, ApJS, 114, 151

Curdt, W., Tian, H., Dwivedi, B. N., \& Marsch, E. 2008, A\&A, 491, L13

Dammasch, I. E., Curdt, W., Dwivedi, B. N., \& Parenti, S. 2008, AnGeo, 26, 2955

Dere, K. P., Landi, E., Young, P. R., et al. 2009, A\&A, 498, 915

Doschek, G. A., Vanhoosier, M. E., Bartoe, J.-D. F., \& Feldman, U. 1976, ApJS, 31,417

Feldman, U., Widing, K. G., \& Warren, H. P. 1999, ApJ, 522, 1133

Foukal, P. V. 1976, ApJ, 210, 575

Galsgaard, K., \& Nordlund, Å. 1996, JGR, 101, 13445

Gudiksen, B. V., Carlsson, M., Hansteen, V. H., et al. 2011, A\&A, 531, A154

Gudiksen, B. V., \& Nordlund, Å. 2005, ApJ, 618, 1020

Hansteen, V. 1993, ApJ, 402, 741

Hansteen, V. H., Carlsson, M., \& Gudiksen, B. 2007, in ASP Conf. Ser. 368, The Physics of Chromospheric Plasmas, ed. P. Heinzel, I. Dorotovič, \& R. J. Rutten (San Francisco, CA: ASP), 107

Hansteen, V. H., \& Gudiksen, B. 2005, in Proc. Solar Wind 11/SOHO 16, Connecting Sun and Heliosphere, ed. B. Fleck, T. H. Zurbuchen, \& H. Lacoste (ESA-SP 592; Noordwijk: ESA), 483

Hansteen, V. H., Hara, H., De Pontieu, B., \& Carlsson, M. 2010, ApJ, 718, 1070

Hayek, W., Asplund, M., Carlsson, M., et al. 2010, A\&A, 517, A49

Hendrix, D. L., van Hoven, G., Mikic, Z., \& Schnack, D. D. 1996, ApJ, 470,1192

Klimchuk, J. A. 2006, SoPh, 234, 41

Mariska, J. T. 1992, The Solar Transition Region (Cambridge: Cambridge Univ. Press)

Martínez-Sykora, J., Hansteen, V., \& Carlsson, M. 2008, ApJ, 679, 871

Martínez-Sykora, J., Hansteen, V., DePontieu, B., \& Carlsson, M. 2009, ApJ, 701,1569

Parker, E. N. 1972, ApJ, 174, 499

Peter, H., Gudiksen, B. V., \& Nordlund, Å. 2006, ApJ, 638, 1086

Peter, H., \& Judge, P. G. 1999, ApJ, 522, 1148

Pneuman, G. W., \& Kopp, R. A. 1977, A\&A, 55, 305

Reale, F. 2010, LRSP, 7, 5

Spadaro, D., Lanza, A. F., Karpen, J. T., \& Antiochos, S. K. 2006, ApJ, 642,579

Zacharias, P., Bingert, S., \& Peter, H. 2009, MmSAI, 80, 654 